Rev. Biol. Trop. 46(4): 1047-1057, 1998

www.ucr.ac.cr www.ots.ac.cr www.ots.duke.edu

\title{
Anatomía y usos de la madera de ocho especies tropicales de Quintana Roo, México
}

\author{
Silvia Rebollar y Alejandra Quintanar
}

Departamento de Biología, Universidad Autónoma Metropolitana-Iztapalapa. A.P. 55-535, 09340 México, D.F. Fax: 724-46-88. Correo electrónico sired@xanum.uam.mx

Recibido 11-IX-1997. Corregido 24-IX-1998. Aceptado 2-X-1998.

\begin{abstract}
This paper describes the wood anatomy of Acacia gaumeri Blake, Diphysa carthagenensis Jacq., Gliricidia sepium (Jacq.) Steud., Leucaena glauca (L.) Benth., Senna racemosa (Mill) Irwin \& Barneby, Murraya paniculata L.Mant., Maytenus guatemalensis Lundell, and Pimenta dioica (L.) Merr. These species are aesthetically attractive as well as hard, highly durable and resistant to insects and locally are widely used in posts, fences, sleepers and rural buildings. Samples were collected from the rainforest in Quintana Roo, Mexico. One tree for each species was collected, and macroscopic and microscopic characteristics were described and measured in wood samples, permanent slides and macerated material. Woods have diffuse porosity, simple perforation plates, alternate and opposite vessel pits and small rays. Crystals are absent only in Acacia gaumeri and Senna racemosa. Axial parenchyma aliform paratracheal, banded types and libriform fibres were common. Differences were found in rays, pore arrangement, presence of fibrotracheids, vascular tracheids and tyloses.
\end{abstract}

Key words: Wood anatomy, trees, tropical rain forest, Quintana Roo, México.

Las selvas de Quintana Roo poseen una riqueza excepcional en sus especies maderables las que se encuentran ampliamente distribuídas; muchas de ellas presentan características anatómicas macroscópicas y microscópicas que repercuten en sus propiedades tecnológicas y por consiguiente en los usos más adecuados a los que se puedan destinar. Esto último ha sido propuesto y discutido para especies pertenecientes a varias familias que conforman la gran diversidad florística representada en las selvas del estado (Rebollar et al.1987, 1993, 1994 y 1996-1997). Así tenemos que la madera estudiada de algunas de las mal llamadas "corrientes" tienen por ejemplo, cualidades anatómicas de porosidad, tamaño de los elementos constitutivos, contenido de extractivos, así como diferencias de color entre albura y duramen que permiten entender los usos que localmente y de forma empírica, les ha asignado la comunidad campesina maya. Por éstas razones se considera preciso contribuir al conocimiento de la anatomía de éste importante grupo de especies en relación con los usos locales que tienen. Se seleccionaron ocho de ellas, pertenecientes a diversas familias que vegetan en la zona norte y sur del estado donde se han caracterizado como duras pesadas y resistentes a la pudrición, a la polilla, humedad y que han sido usadas tradicional y principalmente en la construcción rural, para elaborar durmientes, cercas, postería, tablones y leña. 


\section{MATERIALES Y MÉTODOS}

Las especies estudiadas proceden de una selva mediana subperennifolia que pertenece al Jardín Botánico "Dr. Alfredo Barrera Marín" y Central Vallarta en Puerto Morelos, Municipio de Benito Juárez, y de asociaciones secundarias de una selva alta perennifolia, que pertenece a los ejidos Tres Garantías y Los Ángeles, en el Municipio de Othón P. Blanco, estado de Quintana Roo.

La madera estudiada provino de árboles sanos de fustes rectos con alturas de 8 a 13 $\mathrm{m}$ y diámetros de 20 a $23 \mathrm{~cm}$, aunque son especies que pueden tener diámetros hasta de 40 y $80 \mathrm{~cm}$; la recolección se hizo de acuerdo a las especificaciones de Ramos y Díaz (1981).

El seccionado de las trozas y el muestreo para obtener el material para los estudios macroscópicos y microscópicos se llevó a cabo de acuerdo con la metodología presentada en detalle en Rebollar et al. (1987, 1993).

La denominación de los caracteres macroscópicos se hizo de acuerdo con Tortorelli (1956) y para el color se usaron las tablas de Munsell (1954). Para las descripciones microscópicas se usó la nomenclatura de IAWA Committee (Anónimo 1989) y para los rayos se uso tambien la clasificación de Kribs (1968).

A los elementos mensurables se les hizo un análisis estadístico univariado con un error de muestreo del $5 \%$ y se denominaron con base a la media según la clasificación de Chattaway (1932) y la de IAWA Committee (Anónimo 1937,1939$)$. El valor para el número de poros se da en milímetros cuadrados (mm2), para número de rayos en milímetros lineales $(\mathrm{mm})$, y para el resto de los caracteres en micrones $(\mu \mathrm{m})$.

Las descripciones de las especies se presentan en orden filogenético según la clasificación de Engler modificada por De Dalla Torre y Harms (1963), para cada una se proporcionan: la familia, los nombres comunes, distribución en México, las descripciones anatómicas y los usos locales de la madera que le dan los campesinos mayas.

\section{RESULTADOS}

\section{Acacia gaumeri}

Familia:Leguminosae

Nombres comunes: kanatzin, boxkatzim, katzim (Standley 1961).

Distribución en México: Península de Yucatán (Téllez et al.1982).

Características anatómicas de la madera:

a. Estéticas. La madera presenta diferencia de color entre albura y duramen, la albura es de color amarillo (HUE 10YR 7/6) y el duramen es de color castaño rojizo (HUE 5YR $5 / 6$ ), tiene olor y sabor característicos; su brillo es bajo, de veteado pronunciado, textura gruesa e hilo entrecruzado. Las zonas de crecimiento están marcadas por parénquima axial.

b. Macroscópicas y microscópicas (Figs. 2-4A). Todos los elementos constitutivos son visibles a simple vista. Los poros son de distribución difusa, la mayoría múltiples radiales de 2, algunos solitarios, son muy pocos de 2 (1$3) / \mathrm{mm}^{2}$ y de diámetro tangencial moderadamente pequeño de $69(40-80) \mu \mathrm{m}$. Los elementos de vaso son muy cortos de 213 (186-246) $\mu \mathrm{m}$, sus paredes presentan puntuaciones areoladas alternas y placa perforada simple. El parénquima axial es en bandas, con un ancho de hasta 10 hileras de células. Se presenta estratificado.Los rayos son triseriados 3 (2-4), homogéneos, numerosos de $9(8-11) / \mathrm{mm}^{2}$, extremadamente bajos de 141 (132-147) $\mu \mathrm{m}$ y muy finos de 24 (21-37) $\mu \mathrm{m}$.

Las fibras son de tipo libriforme, extremadamente cortas de 313 (264-320) $\mu \mathrm{m}$, de diámetro mediano de 17 (14-22) $\mu \mathrm{m}$ y de paredes delgadas de 5 (4-6) $\mu \mathrm{m}$.

c. Usos locales. Madera dura, se usa en tablas, durmientes y leña.

\section{Diphysa carthagenensis}

Familia: Leguminosae

Nombres comunes: tsuts tsuts, dzucdzuc, zuzul, xbabalche (Standley \& Steyermark 1946). Distribución en México: Península de Yucatán (Standley \& Steyermark 1946). 

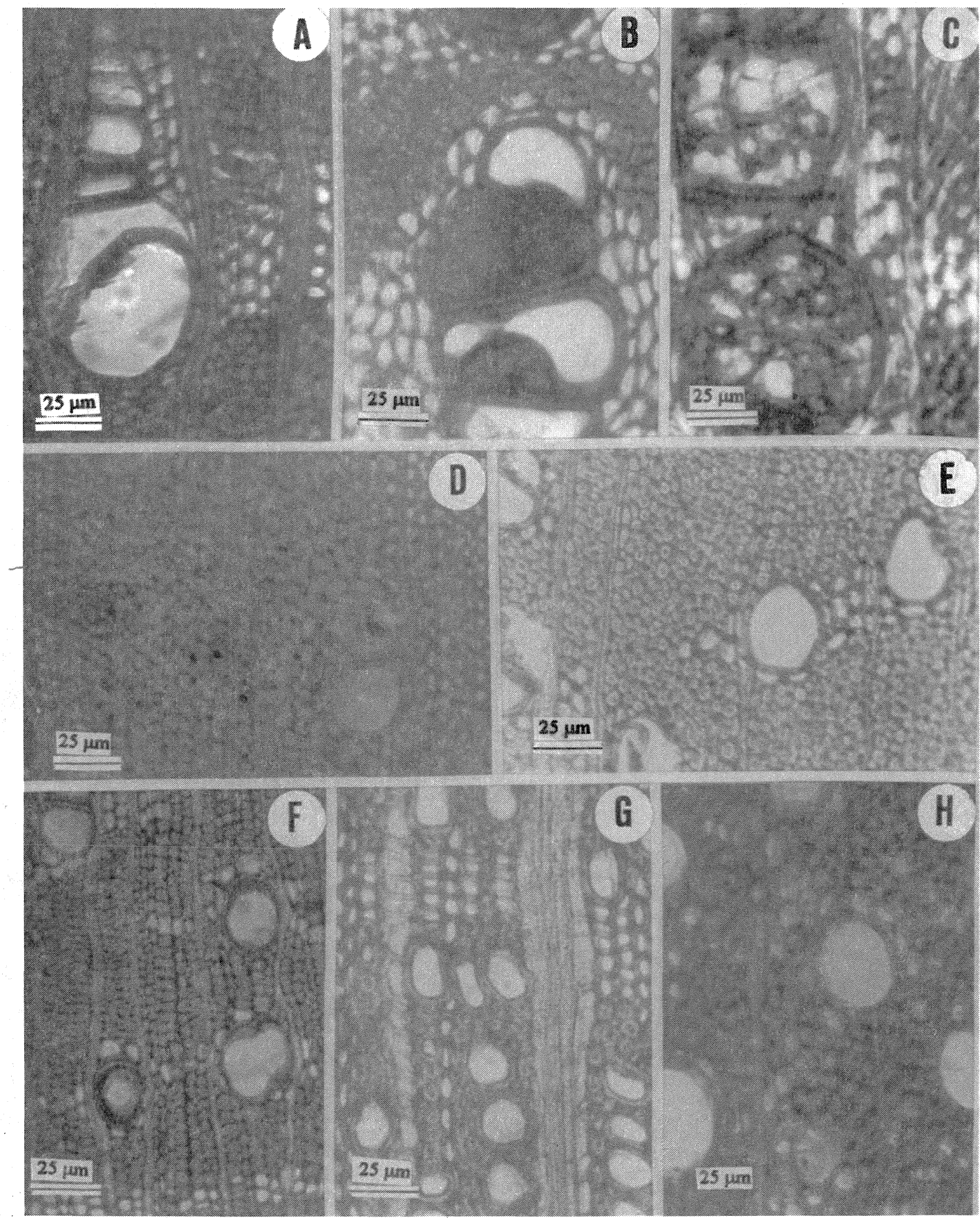

Fig. 1. Cortes transversales de: A. Acacia gaumeri, B. Diphysa carthagenensis, C. Gliricidia sepium, D. Leucaena glauca, E. Senna racemosa F. Murraya paniculata, G. Maytenus guatemalensis, H. Pimenta dioica. 


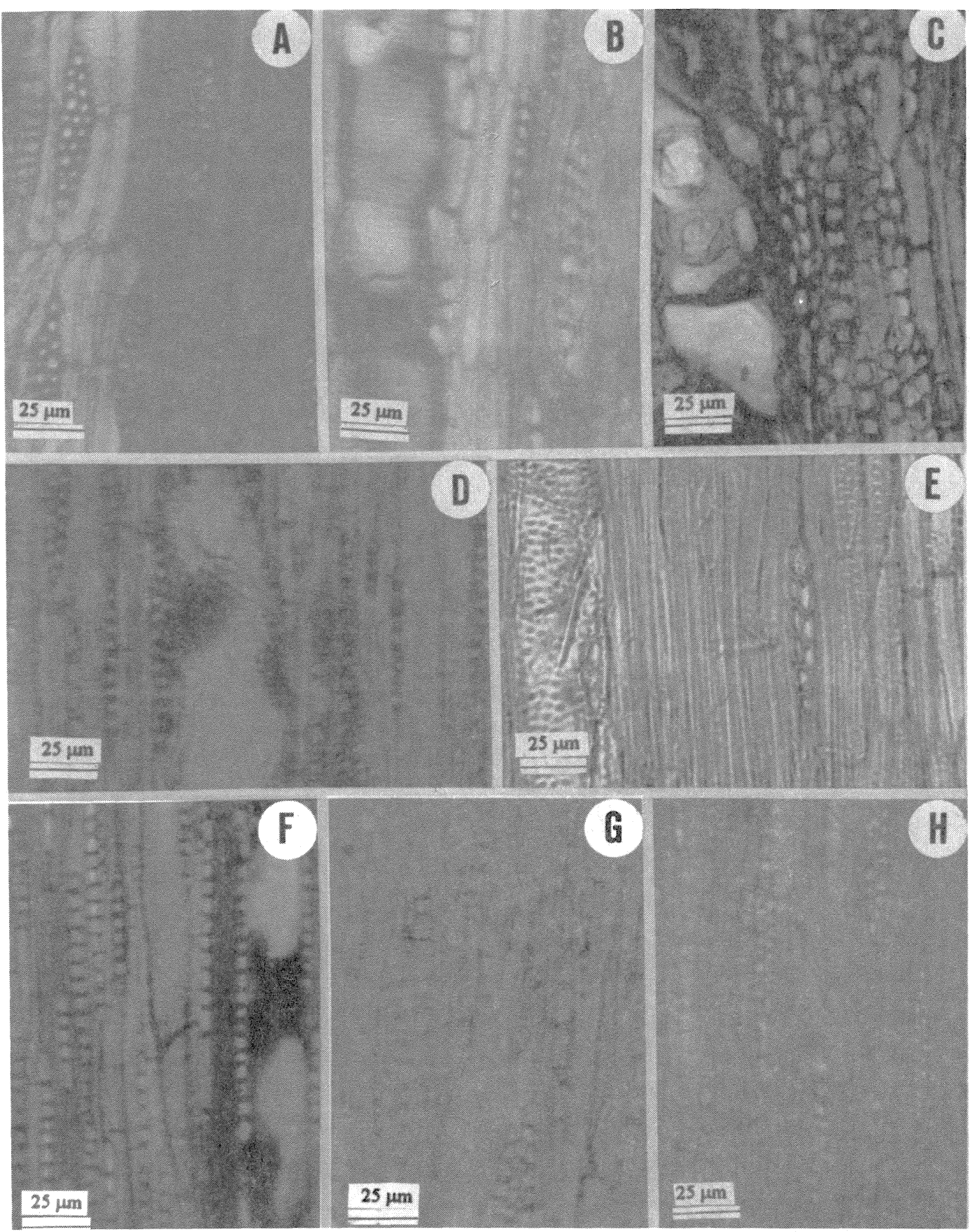

Fig. 2. Cortes tangenciales de: A. Acacia gaumeri, B. Diphysa carthagenensis, C. Gliricidia sepium, D. Leucaena glauca, E. Senna racemosa F. Murraya paniculata, G. Maytenus guatemalensis, H. Pimenta dioica. 

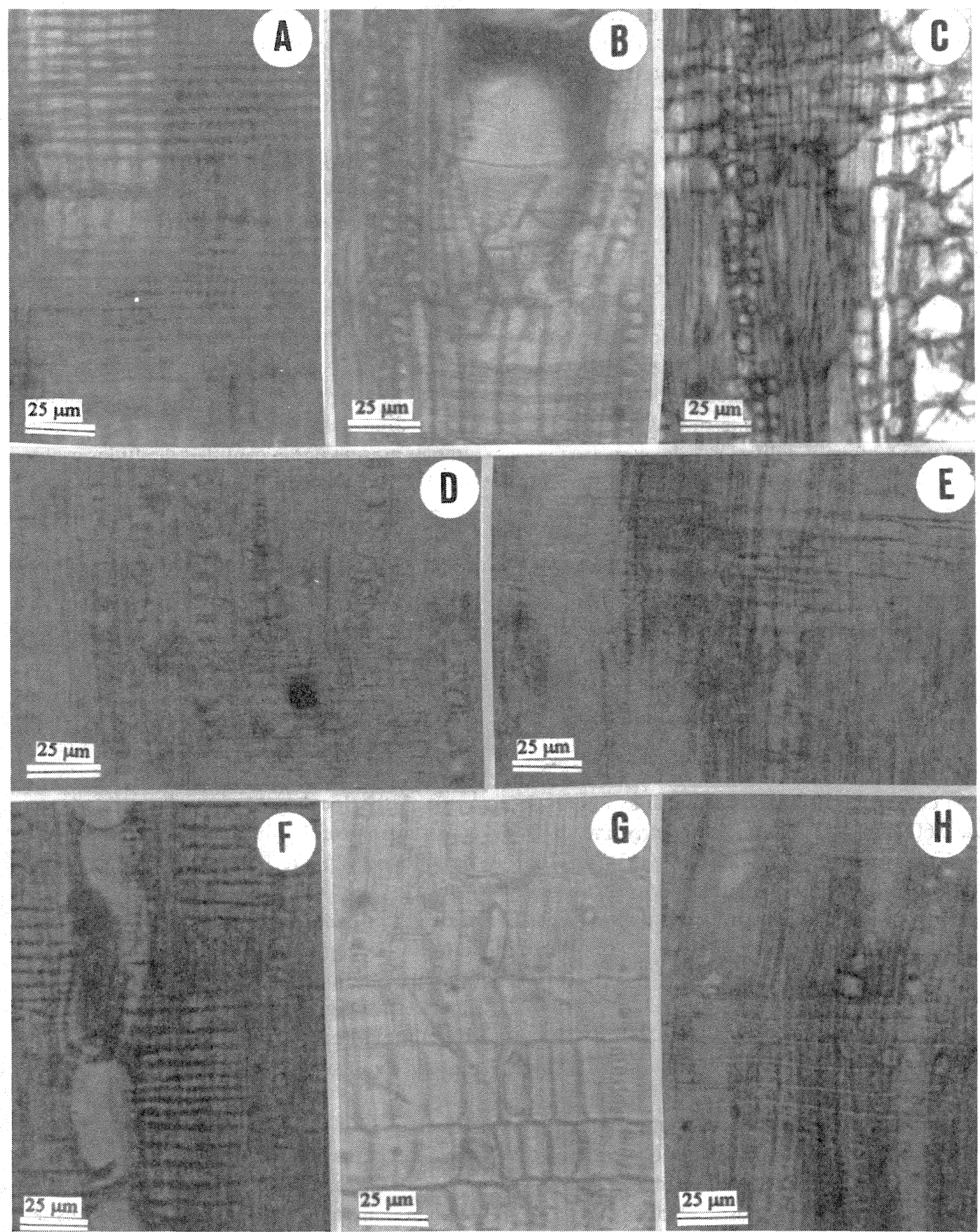

Fig. 3. Cortes radiales de: A. Acacia gaumeri, B. Diphysa carthagenensis, C. Gliricidia sepium, D. Leucaena glauca, E. Senna racemosa F. Murraya paniculata, G. Maytenus guatemalensis, H. Pimenta dioica. 


\section{Características anatómicas de la madera:}

a. Estéticas. La madera presenta diferencias de color entre albura y duramen, la albura es de color castaño muy pálido (HUE 10YR $7 / 3$ ) y el duramen es de color castaño amarillento (HUE 10YR 5/8), no tiene olor característico y el sabor es ligeramente amargo; su brillo es mediano, de veteado pronunciado, textura mediana e hilo entrecruzado. Las zonas de crecimiento marcadas por parénquima axial.

b. Macroscópicas y microscópicas (Figs. 2-4B). El parénquima axial y los rayos son visibles a simple vista y los poros con lupa. Los poros son de distribución difusa, la mayoría solitarios, múltiples radiales de 2 a 3 y agrupados, son moderadamente pocos de 10 (7$15) / \mathrm{mm} 2$, algunos solitarios y de diámetro tangencial mediano de 149 (102-221) $\mu \mathrm{m}$. Los elementos de vaso son cortos de 200 (150-250) $\mu \mathrm{m}$, sus paredes presentan puntuaciones areoladas alternas y placa perforada simple. El parénquima axial es aliforme y aliforme confluente, difuso en bandas y marginal. Presenta cristales de forma romboidal. Los rayos son triseriados 3 (1-3), heterogéneos tipo II, moderadamente numerosos 6 (3-9)/mm, extremadamente bajos 380 (230-490) $\mu \mathrm{m}$ y extremadamente finos de $110(90-150) \mu \mathrm{m}$. Las fibras son de tipo libriforme, extremadamente cortas de 360 (252-513) $\mu \mathrm{m}$, de diámetro fino de 5 (4-7) $\mu \mathrm{m}$, paredes delgadas de 2 (1-3) $\mu \mathrm{m}$.

c. Usos locales. Madera dura, se usa en postes de palapas, construcción rural y leña, resiste la humedad.

\section{Gliricidia sepium}

Familia: Leguminosae

Nombres comunes: sak jab, xak-yaab, sacyab (Standley 1961).

Distribución en México: Vertiente del Golfo, desde Tamaulipas, San Luis Potosi y el norte de Puebla y Veracruz hasta la Península de Yucatán y desde Sinaloa hasta Chiapas en el Pacífico (Pennington \& Sarukhán 1968).

\section{Características anatómicas de la madera:}

a. Estéticas. La madera presenta diferencia de color entre albura y duramen, la albura es de color amarillo (HUE 10YR 7/8) y el duramen es de color castaño obscuro (HUE 7.5YR 3/2), tiene olor y sabor ligeramente aceitosos; su brillo es mediano, de veteado pronunciado, textura mediana e hilo recto. Las zonas de crecimiento están marcadas por el parénquima axial.

b. Macroscópicas y microscópicas. (Figs. 2-4C). Los poros y el parénquima axial son visibles a simple vista y los rayos con lupa. Los poros son de distribución difusa, la mayoría múltiples radiales de 2 a 5 y algunos solitarios, pocos de 4 (1-5)/mm2 y de diámetro tangencial mediano de 130 (75-232) $\mu \mathrm{m}$. Los elementos de vaso son muy cortos de 200 (145-326) $\mu \mathrm{m}$, sus paredes presentan puntuaciones areoladas opuestas y placa perforada simple, presenta gran cantidad de tílides esclerosadas. El parénquima axial es de tipo aliforme y aliforme confluente. Presenta cristales romboidales y gomas. Los rayos son la mayoría uniseriados y multiseriados de 3 (2-5), heterogéneos de tipo I, numerosos de 7 (5$10) / \mathrm{mm}$, extremadamente bajos de 260 (145435) $\mu \mathrm{m}$ y moderadamente finos de 36 (20-52) $\mu \mathrm{m}$. Presentan abundantes cristales. Las fibras son de tipo libriforme, medianas de 1119 (8551522) $\mu \mathrm{m}$, de diámetro mediano 17 (12-24) $\mu \mathrm{m}$ y de paredes gruesas $6(2-10) \mu \mathrm{m}$.

c. Usos locales. Madera dura, se usa en construcción rural y durmientes, no le penetra la polilla ni se pudre.

\section{Leucaena glauca}

Familia: Leguminosae.

Nombres comunes: Waxim, xaxim, huatsin, uaxim (Pennington \& Sarukhán 1968).

Distribución en México: Vertiente del Golfo hasta la Península de Yucatán y en el Pacífico desde Sinaloa hasta Chiapas (Pennington \& Sarukhán 1968).

Características anatómicas de la madera:

a. Estéticas. La madera presenta diferencia de color entre albura y duramen, la albura es de color amarillo (HUE 10YR 8/8) y el düramen es de color castaño fuerte (HUE 7.5YR), no tiene olor y sabor característicos; su brillo es alto, de veteado pronunciado, textura mediana e hilo 
entrecruzado. Las zonas de crecimiento están marcadas por parénquima marginal y fibras.

b. Macroscópicas y microscópicas (Figs. 2-4D). Los poros y el parénquima axial son visibles a simple vista y los rayos con lupa. Los poros son de distribución difusa, la mayoría solitarios, algunos agregados hasta de 6 y escasamente múltiples radiales de 2 y 3 , moderadamente pocos de $9(4-10) / \mathrm{mm}^{2}$ y de diámetro tangencial moderadamente pequeño de 96 (52150) $\mu \mathrm{m}$. Los elementos de vaso son muy cortos de 288 (188-413) $\mu \mathrm{m}$, sus paredes presentan puntuaciones areoladas alternas y placa perforada simple. Presentan gomas y taninos. El parénquima axial es de tipo aliforme y marginal. Presenta cristales romboidales. Los rayos son la mayoría uniseriados biseriados y escasos multiseriados 1 (1-3), todos son homogéneos, moderadamente numerosos $6(4-11) / \mathrm{mm}$, extremadamente bajos de 188 (87-290) $\mu \mathrm{m}$, muy finos de 20 (14-32) $\mu \mathrm{m}$. Las fibras son de tipo libriforme y fibrotraqueidas, moderadamente cortas 787 (507-1015) $\mu \mathrm{m}$, de diámetro fino 11 (8-16) $\mu \mathrm{m}$ y de paredes gruesas 4 (2-6) $\mu \mathrm{m}$. Las fibrotraqueidas presentan gomas.

c. Usos locales. Madera dura, se usa en empalizadas de casas, en postes y tablas, no le penetra la polilla.

\section{Senna racemosa}

Familia: Leguminosae

Nombres comunes: xcanlol, kantumbu (Standley 1924).

Distribución en México: Veracruz, Oaxaca, Chiapas y Península de Yucatán (Standley 1924).

Características anatómicas de la madera:

a. Estéticas. La madera presenta diferencia de color entre albura y duramen, la albura es de color castaño amarillo claro (HUE 10YR 6/4) y el duramen es de color castaño amarillo obscuro (HUE 10YR 4/4), no tiene olor y sabor característicos, su brillo es mediano, de veteado pronunciado, textura fina e hilo entrecruzado. Las zonas de crecimiento marcadas por parénquima axial.

b. Macroscópicas y microscópicas (Figs. 2-4E). Todos los elementos constitutivos son visibles con lupa. Los poros son de distribución difusa, la mayoría solitarios y algunos múltiples radiales de 2 a 3 , son numerosos 18 $(15-21) / \mathrm{mm}^{2}$ y de diámetro tangencial muy pequeño 66 (40-80) $\mu \mathrm{m}$. Los elementos de vaso son muy cortos 210 (190-260) $\mu \mathrm{m}$, sus paredes presentan puntuaciones areoladas alternas y placa perforada simple. El parénquima axial es de tipo aliforme y aliforme confluente. Los rayos son biseriados, homogéneos, son numerosos 8 $(5-11) / \mathrm{mm}^{2}$, extremadamente bajos 120 (90170) $\mu \mathrm{m}$ y extremadamente finos 10 (7-3) $\mu \mathrm{m}$. Las fibras son de tipo libriforme, medianas de $1110(810) \mu \mathrm{m}$, de diámetro mediano de 16 (1020) $\mu \mathrm{m}$ y de paredes gruesas de $6(2-7) \mu \mathrm{m}$.

c. Usos locales. Madera dura, se usa en durmientes, postes de casas, en cercas y como sustituto del guayacán.

\section{Murraya paniculata}

Familia: Rutaceae

Nombres comunes: limonaria, mirto (Standley \& Steyermarck, 1949).

Distribución en México: Yucatán y Quintana Roo (Standley \& Steyermarck 1949).

\section{Características anatómicas de la madera:}

a. Estéticas. La madera presenta diferencias de color entre albura y duramen, la albura es de color amarillo rojizo (HUE 7.5YR 7/8) y el duramen es de color castaño (HUE 7.5YR) con algunas vetas de color castaño fuerte (HUE 7.5YR 5/6), no tiene olor ni sabor característicos, su brillo es mediano, de veteado pronunciado, textura mediana e hilo entrecruzado. Las zonas de crecimiento poco marcadas por el parénquima marginal.

b. Macroscópicas y microscópicas (Figs. 2-4F). Todos los elementos constitutivos son visibles con lupa. Los poros son de distribución difusa, la mayoría múltiples radiales de 2 a 4 , escasamente solitarios, son numerosos de 16 (10-19)/ $\mathrm{mm}^{2}$, de diámetro tangencial moderadamente pequeño de $56(40-74) \mu \mathrm{m}$. Los elementos de vaso son medianos de 367 (181-500) $\mu \mathrm{m}$, sus paredes con puntuaciones areoladas alternas y placa perforada simple. Presentan gomas y posiblemente taninos. El parénquima axial es aliforme confluente de hasta 3 hileras 
de células y marginal. Presenta cristales. Los rayos son uniseriados, homogéneos, muy numerosos $13(9-17) / \mathrm{mm}$, extremadamente bajos de $271(108-507) \mu \mathrm{m}$ y muy finos 16 (8-24) $\mu \mathrm{m}$. Presentan taninos. Las fibras son de tipo libriforme y fibrotraqueidas, moderadamente cortas 799 (580-1065) $\mu \mathrm{m}$, diámetro fino 11 (6-16) $\mu \mathrm{m}$ y de paredes gruesas 4 (2-7) $\mu \mathrm{m}$.

c. Usos locales. Madera dura, se usa para postes y construcción rural.

\section{Maytenus guatemalensis.}

Familia: Celastraceae

Nombres comunes: chak che' (datos de colecta). Distribución en México: Península de Yucatán (Standley \& Steyermark 1949).

Características anatómicas de la madera:

a. Estéticas. La madera presenta diferencia de color entre albura y duramen, la albura es de color castaño rojizo claro (HUE 5YR 5/4 ) y el duramen es de color castaño rojizo (HUE 5YR 4/4 ) con veteados rojizos, no tiene olor ni sabor característicos, su brillo es mediano, de veteado pronunciado, textura fina e hilo entrecruzado. Las zonas de crecimiento están marcadas por parénquima axial.

b. Macroscópicas y microscópicas (Figs. 2-4G). Todos los elementos constitutivos son visibles a simple vista. Los poros son de distribución difusa, exclusivamente solitarios, muy numerosos de 46 (38-52)/ $/ \mathrm{mm}^{2}$ y de diámetro tangencial extremadamente pequeño de 26 (18-30) $\mu \mathrm{m}$. Los elementos de vaso son extremadamente cortos de 162 (141-216) $\mu \mathrm{m}$, sus paredes presentan puntuaciones areoladas opuestas y placa perforada simple. El parénquima axial es bandeado confluente de 4 hasta 6 hileras de células de ancho. Presenta cristales romboidales. Los rayos son triseriados $3(2-4)$, heterogéneos tipo II, numerosos de $9(7-12) / \mathrm{mm}$, extremadamente bajos de 196 (138-243) $\mu \mathrm{m}$ y moderadamente finos de 36 (20-52) $\mu \mathrm{m}$. Las fibras son de tipo libriforme, medianas $921(829-1011) \mu \mathrm{m}$, de diámetro mediano de $19(18-20) \mu \mathrm{m}$ y de paredes gruesas de 8 (7-10) $\mu \mathrm{m}$.

c. Usos locales. Madera dura, se usa en construcción rural, para tablas y leña resistente a la humedad.

\section{Pimenta dioica.}

Familia: Myrtaceae.

Nombres comunes: pimienta, pimienta gorda (Mc Vaugh 1963).

Distribución en México: Se encuentra restringida a la vertiente del Golfo desde el norte de Puebla y Veracruz hasta el sur de la Península de Yucatán (Pennington \& Sarukhán 1968).

\section{Características anatómicas de la madera:}

a. Estéticas. La madera es de color amarillo (10 YR 8/6), no tiene olor ni sabor característicos, su brillo es bajo, de veteado liso, textura fina e hilo recto. Las zonas de crecimiento no están marcadas.

b. Macroscópicas y microscópicas (Figs. 2-4H). Todos los elementos constitutivos visibles con lupa. Los poros son de distribución difusa, exclusivamente solitarios, son numerosos $23(20-29) / \mathrm{mm}^{2}$, de diámetro tangencial muy pequeño de 30 (24-33) $\mu \mathrm{m}$. Los elementos de vaso son muy cortos de $210(120-240) \mu \mathrm{m}$, rodeados por traqueidas vasicéntricas rectangulares, sus paredes presentan puntuaciones areoladas alternas y placa perforada simple. El parénquima axial es en bandas y reticulado con una sola hilera de células. Presenta cristales romboidales. Los rayos son uniseriados y multiseriados de 1 (1-3), heterogéneos tipo I, numerosos de $9(8-10) / \mathrm{mm}$, extremadamente bajos 219 (135-294) $\mu \mathrm{m}$, muy finos 18 (15-21) $\mu \mathrm{m}$. Presentan gomas. Las fibras son de tipo libriforme, medianas de 1490 (880-1664) $\mu \mathrm{m}$, de diámetro mediano de $22(18-30) \mu \mathrm{m}$ y de paredes muy gruesas de 9 (6-10) $\mu \mathrm{m}$.

c. Usos locales. Madera dura, se usa en construcción rural.

\section{DISCUSIÓN}

La madera de la mayoría de las especies presentó diferencia de color entre la albura y el duramen y veteado pronunciado, a excepción de Pimenta dioica que es amarilla y con veteado liso, ésta diversidad de tonalidades y veteados así como el agradable olor de $A$. gaumeri y el brillo que presentan, las hacen tener un alto valor estético para ser usadas en decoración de 
interiores, lambrín, muebles finos y pisos. La textura fina de $S$. racemosa, $M$. guatemalensis y $P$. dioica, las hace adecuadas para elaborar artículos torneados y esculturas. El hilo recto de G. sepium y de $P$. dioica las hace resistentes al impacto, por lo que se pueden usar en partes de instrumentos musicales, mangos para herramientas, artículos deportivos, pisos, y escaleras. A. gaumeri, D. carthagenensis, L. glauca, $S$. racemosa, $M$. paniculata y $M$. guatemalensis, tienen hilo entrecruzado, propiedad que permite retener bien los clavos y tornillos en ensambles machimbrados, también les da resistencia , cualidades óptimas para usos en los que se requiere resistencia mecánica y al desgaste (De la Paz Pérez \& Carmona 1979), por lo que también son adecuadas para usarse en construcciones pesadas, en barcos, puentes, muelles, pilotes, en partes de maquinaria pesada como lanzaderas para la industria textil.

La porosidad difusa y las dimensiones pequeñas de vasos y rayos, presentes en todas las especies puede explicar en buena parte respuestas positivas al alto impacto, así como las paredes gruesas de las fibras que a excepción de $D$. carthagenensis, son características que repercuten en las propiedades físicas de contracciones volumétricas, y en las mecánicas de compresión paralela y perpendicular, lo que justifica los usos propuestos. Otro aspecto importante es la presencia de cristales, gomas y taninos en casi todas las especies, en particular para G. sepium que además presenta tílides comunes y esclerosadas, lo que garantiza un buen comportamiento en usos donde se requiere alta durabilidad natural.

Es interesante resaltar las caraterísticas anatómicas encontradas para otras especies de leguminosas, como la presencia del parénquima axial aliforme y aliforme confluente, marginal y en bandas, así como la estructura estratificada de $A$. gaumeri lo que coincide con lo estudiado por Cárdenas (1971). En cuanto a las características macroscópicas y microscópicas cabe resaltar al parénquima axial y radial, los contenidos celulares, tílides fibrotraqueidas y traqueidas vasicéntricas como caracteres distintivos de las especies.
Por lo anterior estas especies son de gran importancia, además por su abundancia en las selvas de Quintana Roo (Téllez et al.1982, Escalante 1986,), sobre todo en aquellas zonas perturbadas por la extracción de madera o por cultivos ocasionales en asociaciones secundarias (acahuales o huimiles altos) que son más ricos en especies que la selva de donde derivaron, tal es el caso de G. sepium y $L$. glauca que además tienen un rápido crecimiento y se reproducen por estacas (Fors 1975).

Es importante señalar que en el estado de Yucatán, A. gaumeri, D. carthagenensis, G. sepium y $S$. racemosa, son especies abundantes en el monte de $\mathrm{X}$ huilub, en una vegetación secundaria de selva mediana subperennifolia (Sánchez 1993) y sólo son usadas para leña. Otro ejemplo es el de A. gaumeri, G. sepium y $S$. racemosa, especies que forman parte importante de los huertos familiares en $\mathrm{X}^{\prime}$ huilub, Yuc., que también son usadas como combustible y ocasionalmente en construcción, Herrera (1994).

\section{AGRADECIMIENTOS}

Las autoras agradecen al Equipo Técnico del Jardín Botánico "Dr. Alfredo Barrera Marín" en Puerto Morelos, Municipio de Benito Juárez, y a los Ejidos Productores Forestales de la Zona Sur, S.C., en Tres Garantías, Municipio de Othón P. Blanco del estado de Quintana Roo, en especial a Odilón Sánchez y Honorato Huitzil, a Alfonso Argüelles y Mateo Poot, respectivamente. por las facilidades brindadas en la selección y recolección de las especies. Al Departamento de Productos Forestales y Conservación de Bosques del Instituto de Ecología, A.C., por el procesado de la madera, a Ingrid Olmsted y Rafael Durán por la determinación botánica de las especies. A Carmen de la Paz Pérez, y Blanca Pérez, por la revisión y valiosas sugerencias al manuscrito. Este trabajo fue financiado por la Secretaría de Educación Pública a través de los Convenios 089-01-0247, Registro DGICSA 911951. 


\section{RESUMEN}

Se estudió la anatomía de la madera de ocho especies procedentes de asociaciones secundarias de las selvas alta y mediana subperennifolia del estado de Quintana Roo: Acacia gaumeri, Diphysa carthagenensis Gliricidia sepium, Leucaena glauca, Senna racemosa, Murraya paniculata, Maytenus guatemalensis, y Pimenta dioica, de acuerdo con la metodología establecida. Son especies de interés por su distribución, abundancia en el estado, y por los usos que tradicionalmente les han dado los campesinos mayas en postería, cercas, construcción rural durmintes, tablones y leña, debido a las cualidades de dureza y resistencia a la humedad y a los insectos. Las especies presentan porosidad difusa, los vasos con puntuaciones areoladas opuestas y alternas, la placa perforada es simple; el parénquima axial se presenta aliforme y en bandas; con cristales en todas las especies a excepción de Acacia gaumeri y Senna racemosa. Los rayos son bajos finos y numerosos; las fibras son de tipo libriforme. Las diferencias encontradas son: el arreglo de los poros, tipo de parénquima axial $\mathrm{y}$ radial, la presencia de fibrotraqueidas y traqueidas vasculares y tílides.

\section{REFERENCIAS}

Anónimo. 1937. Standard terms of size lenghts of vessel members and wood fibres. Trop. Woods 51:21.

Anónimo. 1939. Standars of size for vessel diameter and ray width. Trop. Woods 59: 51-52.

Anónimo. 1954. Munsell soil color charts. Munsell, Baltimore, Maryland. 17 p.

Anónimo. 1989. IAWA list of microscopic features for hardwood identification. IAWA Bulletin n s. 10:219-332.

Cárdenas, E. 1971. Estudio anatómico de la madera de 8 especies de Leguminosas. Tesis de Licenciatura. Escuela Nacional de Ciencias Biológicas. Instituto Politécnico Nacional. México, D.F.

Chattaway, M. 1932. Proposed standards for numerical value used in describing woods. Trop. Woods 29: 20-28.

De Dalla Torre, C. \& H. Harms. 1963. Genera siphonogamarum ad systema Englerianum conscripta. Akademischen Druck-Uberlagsanstalt. Graz. 637 p.

De la PazPérez- O.C.\& T.F. Carmona. 1979. Influencia del hilo en algunas características tecnológicas de la madera. Bol. Téc. Inst. Nac. Invest. For. 60. México, D. F. 46 p.

Escalante, S. 1986. Estructura y composición de la selva mediana subperennifolia presente en el Jardín
Botánico del CIQRO, Puerto Morelos, Quintana Roo. Tesis de Licenciatura. Facultad de Ciencias Biológicas. Universidad Veracruzana. Xalapa, Ver. México.

Fors, A.J. 1975. Maderas Cubanas. Instituto Cubano del Libro y Facultad de Ciencias Agropecuarias Universidad de La Habana, La Habana. .171 p.

Herrera, N.D. 1994. Los huertos familiares mayas en el oriente de Yucatán. 169 p. In: Etnoflora Yucatanense. Universidad Autónoma de Yucatán. Fasc. 9. Mérida, Yuc.

Kribs, D.A. 1968. Commercial foreign woods on the american market. Dover, Nueva York. 241 p.

Mc Vaugh, R. 1963. Myrtaceae. In: Flora of Guatemala. Fieldiana Botany (24): 382-384 Fieldiana Bot. Field Mus. Nat. Chicago.

Pennington, D. \& J. Sarukhán. 1968. Árboles Tropicales de México. Instituto Nacional de Investigaciones Forestales y Organización de las Naciones Unidas para la Agricultura y la Alimentación. México, D.F. 413 p.

Ramos, C. \& V. Díaz. 1981. Instrucciones para colectar muestras de madera para estudios tecnológicos. Bol. Div. Inst. Nac. Invest. For. 54. México, D. F. 15 p.

Rebollar, S., C. de la Paz Pérez-O. \& A. Quintanar. 1987. Maderas de la Península de Yucatán. México. Biótica 12:159-179.

Rebollar, S., C. de la Paz Pérez-O. \& A. Quintanar. 1993. Anatomía de la madera de cinco especies de Quintana Roo, México. Bol. Soc. Bot. Méx. 53: 103-124.

Rebollar, S., A. Quintanar \& C. de la Paz Pérez-O. 1994. Estudio anatómico de la madera de Psidium sartorianum (Myrtaceae) ỳ Cordia gerascanthus (Boraginaceae). Acta Bot. Méx. 27: 89-97.

Rebollar, S., C.de la Paz Pérez-O. \& A. Quintanar. 19961997. Anatomía de la madera de ocho especies de la selva mediana subperennifolia de Quintana Roo, México. Rev. Biol. Trop. 44(3)/45(1): 67-77.

Sánchez, M.C. 1993. Uso y manejo de la leña en X-huilub, Yucatán. 117 p. In: Etnoflora Yucatanense. Universidad Autónoma de Yucatán. Fasc. 8. Mérida, Yucatán.

Standley, P. 1924. Leguminosae. Trees and shrubs of Mexico. Smithsonian Institution. 23: 410-411.

Standley, P. \& J. Steyermark. 1946. Leguminosae. Flora de Guatemala. Field.Bot. 24: 244-245. 
Standley, P. \& J. Steyermark. 1949. Celastraceae. 24:206208. In: Flora of Guatemala. Field. Bot. Field Mus. Nat. Part VI. Chicago.

Standley, P. 1961. Trees and shrubs of Mexico. 23: 377-482. Field Museum of Natural History, Chicago, Illinois.
Téllez, O., M. Sousa \& E. Cabrera. 1982. Imágenes de la Flora Quintanarroense. CIQRO. A.C. Puerto Morelos, Q. Roo. México. 224 p.

Tortorelli, L. 1956. Maderas y bosques argentinos. ACME, Buenos Aires. 910 p. 